\title{
On a formula of Coll-Gerstenhaber-Giaquinto
}

\author{
H.-G. Gräbe \\ A.T. Vlassov* \\ Univ. Leipzig, Germany \\ State University of Belarus
}

Nov 22, 1996

\begin{abstract}
We present a unifying approach to deformations of an associative algebra $A$ that allows to derive known formulas of Moyal-Vey (1949) and Coll-Gerstenhaber-Giaquinto (1989) from a more general point of view. Such universal deformation formulas correspond to special deformations of the comultiplication of a bialgebra.
\end{abstract}

\section{$1 \quad$ Introduction}

Let $K$ be a ring containing the field $\mathbf{Q}$ of rational numbers, $K^{\prime}=K[[h]]$ be the algebra of formal series on $h$ and $\left(A, \mu_{A}\right)$ a $K$-algebra. This algebra structure extends in a natural way by $K^{\prime}$-linearity to the algebra $A^{\prime}:=A[[h]]$ of power series in $h$ with coefficients in $A$ that we will denote by some abuse of notation also by $\mu_{A}$. The aim of this paper is to study deformations of this structure.

Definition $1 A$ (formal) deformation of the $K$-algebra $A$ is an algebra structure $A_{h}=$ $\left(A^{\prime}, \mu_{h}\right)$ on $A^{\prime}$ with

$$
\mu_{h}:=\mu_{A}+\sum_{k=1}^{\infty} h^{k} \varphi_{k}: A^{\prime} \otimes A^{\prime} \rightarrow A^{\prime} .
$$

For $\mu_{h}$ to be associative in first order on $h, \varphi_{1}$ must fulfill the property

$$
\varphi_{1}\left(a_{1} a_{2}, a_{3}\right)+\varphi_{1}\left(a_{1}, a_{2}\right) a_{3}=\varphi_{1}\left(a_{1}, a_{2} a_{3}\right)+a_{1} \varphi_{1}\left(a_{2}, a_{3}\right)
$$

for $a_{1}, a_{2}, a_{3} \in A$, i.e. has to be a 2-cocycle in the Hochschild complex of $A$. Such a 2-cocycle $\varphi_{1}$ is called an infinitesimal of the deformation. We restrict ourselves to the case when the 2-cochains $\varphi_{k}$ have the form $\varphi_{k}=\mu_{A} \circ P_{k}$, where $P_{k}: A \otimes A \rightarrow A \otimes A$ are $K$-linear maps. Given a 2-cocycle $S:=P_{1}$ we try to define $P_{k}$ for $k \geq 2$ so that $\mu_{h}$ is associative.

In practical applications such a 2-cocycle often appears as the product of 1-cocycles $S=D \otimes E$, where $D, E$ are elements of a certain Lie algebra $\mathcal{G}$ acting by derivations on $A$. There are two famous results that describe prolongations of such 2-cocycles to associative multiplications on $A_{h}$ :

\footnotetext{
*Partially supported by the SMWK-grant 4-7531.50-04-0361/614.
} 
Theorem 1 (Moyal-Vey, [7], [3]) If the Abelian Lie algebra $\mathcal{G}$ acts on a $K$-algebra $A$ by derivations, then for any element $S \in \mathcal{G} \otimes \mathcal{G}$ the composition $\mu_{A} \circ S$ is a D-cocycle and the multiplication

$$
\mu_{h}=\mu_{A} \circ e^{h S}
$$

is associative.

Theorem 2 (V.Coll, M.Gerstenhaber, A.Giaquinto, [1]) If the 2-dimensional Lie algebra $\mathcal{G}$ with generators $E, D$ and commutator relation $[E, D]=E$ acts on the $K$-algebra $A$ by derivations, then for $S=E \otimes D$ the composition $\mu_{A} \circ S$ is a 2-cocycle and the multiplication

$$
\mu_{h}=\mu_{A} \circ(1+h E \otimes 1)^{1 \otimes D}
$$

is associative.

Both theorems were first proved by direct calculations. For Moyal-Vey's theorem these computations are straightforward and use only the Leibniz rule, since $D$ and $E$ commute. The second result is less elementary. We will refer to this example as Gerstenhaber's.

Below we present a unifying approach to these results and give some generalizations of the above formulas. It is based on the notion of an admissible bialgebra action on $A$ that allows to derive both results as partial cases of a more general principle to construct algebra deformations. More precisely, we replace the universal enveloping algebra $U_{h}(\mathcal{G})$ of the Lie algebra $\mathcal{G}$ by a bialgebra $B$ and define conditions on an element $P \in(B \otimes B)[[h]]$ such that for any admissible bialgebra action $\rho: B \rightarrow \operatorname{End}_{K}(A)$ the composition $\mu_{h}=\mu_{A} \circ(\rho \otimes \rho)(P)$ defines a deformation of $A$, i.e. we construct universal deformation formulas in the spirit of [5]. It turns out that for a consistent theory deformations of $A$ have to be associated with deformations of the comultiplication of $B$ leaving this way the class of universal enveloping algebras.

Different aspects of such a theory are demonstrated on Gerstenhaber's example. It turns out, that in this case on the deformed bialgebra there is a $h$-independent $K$-bialgebra structure that already exists in the first order deformation.

These investigations were stimulated by several discussions of the second author with R.-O. Buchweitz during a one month stay at the University of Toronto in december 1992 and elaborated further during several visits to the University of Leipzig.

Some of the ideas were considered in the articles [9], [10].

\section{Admissible bialgebra actions on algebras}

Let $\left(B, \mu_{B}, \Delta_{B}\right)$ be a bialgebra as defined, for example, in [2]. Here $\mu_{B}$ denotes the algebra multiplication and $\Delta_{B}: B \longrightarrow B \otimes B$ the comultiplication. We use the standard notion where an integer index of an operator, acting on a tensor product, denotes the tensor cofactor, on which the operator acts. For example,

$$
\begin{gathered}
\Delta_{i}: C^{\otimes n} \rightarrow C^{\otimes(n+1)}: c_{1} \otimes \ldots \otimes c_{n} \mapsto c_{1} \otimes \ldots \otimes c_{i-1} \otimes \Delta\left(c_{i}\right) \otimes c_{i+1} \otimes \ldots \otimes c_{n}, \\
\mu_{A, 23}: A^{\otimes 3} \rightarrow A^{\otimes 2}: a_{1} \otimes a_{2} \otimes a_{3} \mapsto a_{1} \otimes \mu_{A}\left(a_{2} \otimes a_{3}\right)=a_{1} \otimes a_{2} a_{3} .
\end{gathered}
$$


For $b \in B$ we use the Sweedler notation $\Delta(b)=\sum b_{(1)} \otimes b_{(2)}$ and $\Delta_{1} \Delta(b)=\Delta_{2} \Delta(b)=$ $\sum b_{(1)} \otimes b_{(2)} \otimes b_{(3)}$ if we need to exploit their special structure as elements of $B \otimes B$ resp. $B \otimes B \otimes B$.

For a $K$-coalgebra $C$ there is a notion of cohomology groups $H^{n}(K, C)$ as explained e.g. in [6]. They are the homologies of the complex

$$
0 \longrightarrow C \longrightarrow \ldots \stackrel{\delta}{\rightarrow} C^{\otimes k} \longrightarrow \ldots
$$

where for $S \in C^{\otimes k}$ the coboundary formula is defined as

$$
\delta S=1 \otimes S+\sum_{i=1}^{k}(-1)^{i} \Delta_{i} S+(-1)^{k+1} S \otimes 1 .
$$

Especially, a 1-cocycle $X \in C$ fulfills the condition $\Delta(X)=X_{1}+X_{2}$. For a 2-cocycle $S \in C \otimes_{K} C$ we get $\Delta_{2}(S)+S_{23}=\Delta_{1}(S)+S_{12}$.

For any two left modules $\left(M, \nu_{M}\right),\left(N, \nu_{N}\right)$ over the algebra $B$ the tensor product $M \otimes_{K}$ $N$ has a natural structure as left module over the algebra $B$ defined by

$$
\nu_{M \otimes N}: B \otimes(M \otimes N) \stackrel{\Delta_{1}}{\longrightarrow}(B \otimes B) \otimes(M \otimes N) \stackrel{S_{23}}{\longrightarrow}(B \otimes M) \otimes(B \otimes N) \stackrel{\nu_{M} \otimes \nu_{N}}{\longrightarrow} M \otimes N .
$$

If a bialgebra $B$ acts on a $K$-algebra $A$ by $\rho: B \longrightarrow \operatorname{End}_{K}(A)$ we have the natural condition that $\mu_{A}: A \otimes A \longrightarrow A$ is a $B$-module homomorphism, i.e.

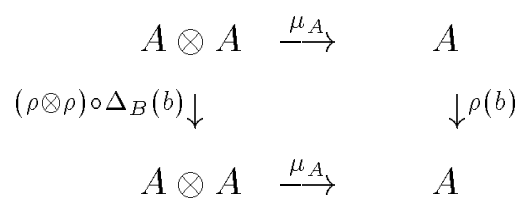

commutes, where $A \otimes A$ is equipped with the $B$-module structure just defined. For $b \in B$ and $a_{1}, a_{2} \in A$ we get the following condition:

$$
\rho_{A}(b)\left(\mu_{A}\left(a_{1} \otimes a_{2}\right)\right)=\mu_{A}\left(\rho_{A \otimes A}(b)\left(a_{1} \otimes a_{2}\right)\right)
$$

or more explicitly

$$
\rho(b)\left(a_{1} \cdot a_{2}\right)=\sum \rho\left(b_{(1)}\right)\left(a_{1}\right) \cdot \rho\left(b_{(2)}\right)\left(a_{2}\right)
$$

This can be written as

$$
\forall b \in B \quad \rho(b) \circ \mu_{A}=\mu_{A} \circ(\rho \otimes \rho) \circ \Delta_{B}(b)
$$

DEFinition $2: A$ homomorphism $\rho: B \rightarrow$ End $_{K}(A)$ of $K$-algebras, that satisfies the compatibility condition (1) between $\mu_{A}$ and $\Delta_{B}$ is called an admissible action of the bialgebra $\left(B, \mu_{B}, \Delta_{B}\right)$ on the $K$-algebra $A$.

For the sake of simplicity we often will omit the symbol of the action $\rho$. Then, for example, (1) has the form

$$
b \circ \mu_{A}=\mu_{A} \circ \Delta_{B}(b) \quad \text { or } \quad b\left(a_{1} a_{2}\right)=\sum b_{(1)}\left(a_{1}\right) b_{(2)}\left(a_{2}\right)
$$


for $a_{1}, a_{2} \in A, b \in B$.

This definition generalizes to bialgebras the concept of actions of universal enveloping algebras induced by Lie algebra of derivations. Indeed, given an algebra $A$ and a Lie algebra $\mathcal{G}$ acting on $A$, the universal enveloping algebra $B=U(\mathcal{G})$ has a natural bialgebra structure with comultiplication $\Delta$ defined by

$$
\Delta(X)=X \otimes 1+1 \otimes X \quad \text { for } X \in \mathcal{G} .
$$

Then the action of $B$ on $A$ is admissible iff for $X \in \mathcal{G}$ and $a_{1}, a_{2} \in A$

$$
X\left(a_{1} \cdot a_{2}\right)=\mu_{A}\left((X \otimes 1+1 \otimes X)\left(a_{1} \otimes a_{2}\right)\right)=\left(X a_{1}\right) a_{2}+a_{1}\left(X a_{2}\right),
$$

i.e. $\mathrm{X}$ is a derivation of $A$.

Note that an action of a bialgebra $B$ on a $K$-algebra $A$ is defined by the action of the generators of $B$ on the generators of $A$.

Examples:

1. The left action of $A$ on itself is an admissible bialgebra action, if we define $\Delta\left(L_{a}\right)=$ $L_{a} \otimes 1$ for the left action $L_{a} \in \operatorname{End}_{K}(A)$ of $a \in A$. Analogously the right action of $A^{\text {op }}$ on $A$ is an admissible bialgebra action wrt. $\Delta\left(R_{a}\right)=1 \otimes R_{a}$.

This may be extended to an admissible action of the enveloping algebra $A^{e}:=A \otimes_{K} A^{o p}$ on $A$, where the comultiplication is given by the rule $\Delta(x \otimes y)=(x \otimes 1) \otimes(1 \otimes y)$. If $A^{e}=\operatorname{End}_{K}(A)$, e.g. for a matrix algebra $M_{n}(K)$, this construction allows to introduce an admissible bialgebra structure on the whole algebra of endomorphisms $\operatorname{End}_{K}(A)$.

2. The natural action of the bialgebra $B=K\left[\frac{\partial}{\partial x_{1}}, \ldots, \frac{\partial}{\partial x_{n}}\right]$ on $A=K\left[x_{1}, \ldots, x_{n}\right]$ is an admissible bialgebra action, since $B$ is the universal enveloping algebra of an Abelian Lie algebra acting on $A$ by derivations.

3. It induces an action of the Weyl algebra $W=A \otimes_{K} B$ on $A$ that is an admissible bialgebra action on $A$ with respect to the natural bialgebra structure on $W$ obtained by scalar extension $K \longrightarrow A$ from $B$. More precisely, the multiplication on $W$ is induced by the commutation rules

$$
\frac{\partial}{\partial x_{i}} \cdot x_{j}=\delta_{i j}+x_{j} \cdot \frac{\partial}{\partial x_{i}}
$$

and the comultiplication by the corresponding rules on $A$ and $B$

$$
\Delta\left(x_{i}\right)=x_{i} \otimes 1 \quad \text { and } \quad \Delta\left(\frac{\partial}{\partial x_{i}}\right)=\frac{\partial}{\partial x_{i}} \otimes 1+1 \otimes \frac{\partial}{\partial x_{i}} .
$$

4. This may be generalized to arbitrary Lie algebras $\mathcal{G}$ acting on $A$ by derivations. Indeed, the admissible bialgebra action of the universal enveloping algebra $B=U(\mathcal{G})$ on $A$ described above may be extended to an admissible bialgebra action on $W=A \otimes_{K} B=A[\mathcal{G}]$ on $A$, if we extend multiplication and comultiplication on $W$ by the following rules:

$$
X \cdot a=X(a)+a \cdot X, \quad \Delta(a)=a \otimes 1, \quad \Delta(X)=X \otimes 1+1 \otimes X .
$$

Here and below $a \in A$ and $X \in \mathcal{G}$ are identified with their images in $W$ under the embeddings $A \rightarrow A \otimes 1 \subset W$ and $\mathcal{G} \rightarrow 1 \otimes \mathcal{G} \subset W$.

5. This may be generalized once more: For any admissible action of a bialgebra $B$ on an algebra $A$ there is a natural bialgebra structure on the $K$-module $W:=A^{e} \otimes B$ 
extending those of $A^{e}$ and $B$. As above we have only to define the product $b \cdot(x \otimes y)$ for $b \in B,(x \otimes y) \in A^{e}$. As easily seen the correct rule is

$$
b \cdot(x \otimes y)=\sum\left(b_{(1)}(x) \otimes b_{(3)}(y)\right) \cdot b_{(2)},
$$

where $\Delta_{1} \Delta(b)=\Delta_{2} \Delta(b)=\sum b_{(1)} \otimes b_{(2)} \otimes b_{(3)}$ are obtained from the comultiplication rule on $B$. Again admissibility of the $B$-action on $A$ guarantees that the given rules define a bialgebra structure on $W$ and the natural action of $W$ on $A$ is admissible.

REMARK: The only place we found in the literature, where a condition similar to (1) was considered, is the following general result [5, Lemma 9.2.]:

Let $V$ be any vector space, $\mu: k\langle V\rangle \otimes k\langle V\rangle \longrightarrow k\langle V\rangle$ be the multiplication in the free (=tensor) algebra $k\langle V\rangle, \mathcal{C} \subset \operatorname{End}(k\langle V\rangle)$ be a subspace of the linear endomorphisms, and $\Delta: \mathcal{C} \longrightarrow \mathcal{C} \otimes \mathcal{C}$ be a linear map such that $\mu \Delta f(a \otimes b)=$ $f(a b)$ for all $f \in \mathcal{C}, a, b \in k\langle V\rangle$. Then $\Delta$ is coassociative, i.e. $\mathcal{C}$ is a coalgebra.

If moreover $C$ is a subalgebra of $\operatorname{End}(k\langle V\rangle)$ and $\Delta$ a $k$-algebra homomorphism the natural action of the bialgebra $\left(\mathcal{C}, \mu_{C}, \Delta\right)$ on $k\langle V\rangle$ is admissible.

\section{Deformations of algebras with an admissible bialgebra ac- tion}

The main idea of this section is the observation that for both formulae considered in the introduction the deformed multiplication has the form $\mu_{h}=\mu_{A} \circ P$ for a certain element $P \in(U(\mathcal{G}) \otimes U(\mathcal{G}))[[h]]$.

So let $B$ be a bialgebra with an admissible action on $A$ as in the last section. As above the bialgebra structure extends to $B^{\prime}=B[[h]]$ by $K^{\prime}$-linearity in such a way that $\left(B^{\prime}, \mu_{B}, \Delta_{B}\right)$ acts admissible on $\left(A^{\prime}, \mu_{A}\right)$. Below we consider the question, how deformations of the algebra structure on $A$ are related to those of the bialgebra $B$.

Let's consider the condition that must be fulfilled by an element $P=1+\sum_{i=1}^{\infty} h^{i} P_{(i)} \in$ $B^{\prime} \otimes_{K^{\prime}} B^{\prime}=\left(B \otimes_{K} B\right)[[h]]$ for $\mu_{h}=\mu_{A} \circ P$ to be associative.

$$
0=\mu_{h} \circ\left(\mu_{h, 12}-\mu_{h, 23}\right)=\mu \circ P \circ\left(\mu_{12} \circ P_{12}-\mu_{23} \circ P_{23}\right)
$$

Since $B$ acts admissible we get $P \circ \mu_{12}=\mu_{12} \circ \Delta_{1}(P), P \circ \mu_{23}=\mu_{23} \circ \Delta_{2}(P)$ and altogether

$$
0=\mu \circ \mu_{12} \circ\left(\Delta_{1}(P) P_{12}-\Delta_{2}(P) P_{23}\right)
$$

Hence

$$
\Delta_{1}(P) P_{12}-\Delta_{2}(P) P_{23}=0
$$

is a sufficient condition for $P$ to make $\mu_{h}$ associative for any admissible $B$-action on $A$.

This yields already a proof of the following generalization of the Moyal-Vey formula.

Theorem 3 If the commutative bialgebra $B$ acts admissible on $A$ then for any 2-cocycle $S \in B \otimes B$ the multiplication

$$
\mu_{h}=\mu_{A} \circ e^{h S}
$$

is associative. 
Proof: Indeed, for $P=e^{h S}$ condition (2) is equivalent to

$$
e^{h \Delta_{1}(S)} \circ e^{h S_{12}}=e^{h \Delta_{2}(S)} \circ e^{h S_{23}}
$$

and finally to

$$
\Delta_{1}(S)+S_{12}=\Delta_{2}(S)+S_{23} .
$$

EXAMPle : Let us consider the commutative bialgebra $B$ with the free generators $E_{i}, D^{i}, L_{i}^{j} \in C, i, j=1, \ldots, n$ and the comultiplication that using the matrix notation

$$
\mathbf{E}=\left(\begin{array}{llll}
E_{1} & E_{2} & \ldots & E_{n}
\end{array}\right), \mathbf{D}=\left(\begin{array}{c}
D^{1} \\
D^{2} \\
\vdots \\
D^{n}
\end{array}\right), \mathbf{L}=\left(L_{i}^{j}\right),
$$

may be written in the following form

$$
\Delta(\mathbf{E})=\mathbf{E}_{1} \mathbf{L}_{2}+\mathbf{E}_{2}, \Delta(\mathbf{D})=\mathbf{D}_{2}+\mathbf{L}_{1} \mathbf{D}_{2}, \Delta(\mathbf{L})=\mathbf{L}_{1} \mathbf{L}_{2} .
$$

Then the 2-cochain $S=\mathbf{E}_{1} \mathbf{D}_{2}=\sum_{i=1}^{n} E_{i} \otimes D^{i}$ is a cocycle and the power series $P=e^{h S}$ satisfies the equation (2).

If $B$ acts admissible on an algebra $A$ this yields an explicit formula for a deformation of $A$ that doesn't fit into the frame of theorem 1.

The solution $P=e^{h S}$ of (2) described in theorem 3 is expressed as an exponential function. Since $f(x, h)=e^{h x}$ is the solution of the differential equation $\frac{\partial f}{\partial h}=x \cdot f$ with initial condition $f(x, 0)=1$ the expression

$$
S_{h}:=P^{-1} \frac{\partial P}{\partial h} \in(B \otimes B)[[h]]
$$

also may play a crucial role for other applications. Note that the power series $P$ is uniquely defined by $S_{h}$ but their connection may be more difficult to describe than in theorem 3 . Since $\left.S_{h}\right|_{h=0}=P_{(1)}$ coincides with the element $S \in B \otimes B$ defined in the introduction, $S_{h}$ is a deformation of $S$ (in a sense to be specified).

Under certain additional assumptions the condition (2) may be reformulated as a condition on $S_{h}$. For example, if $S=S_{h}$ does not depend on $h$ we get $P=\exp (h S)$ and (2) may be reformulated as

$$
e^{h \Delta_{1}(S)} \cdot e^{h S_{12}}-e^{h \Delta_{2}(S)} \cdot e^{h S_{23}}=0 .
$$

If the exponents mutually commute, i.e. $\left[\Delta_{1}(S), S_{12}\right]=\left[\Delta_{2}(S), S_{23}\right]=0$ we can rewrite this equation as

$$
e^{h\left(\Delta_{1}(S)+S_{12}\right)}-e^{h\left(\Delta_{2}(S)+S_{23}\right)}=0
$$

that holds for any 2-cocycle $S$. Thus we proved the following generalization of the previous theorem.

TheOREM 4 Let $S$ be a 2-cocycle of a (of a not necessarily commutative) bialgebra $B$ and

$$
\left[\Delta_{1}(S), S_{12}\right]=\left[\Delta_{2}(S), S_{23}\right]=0 .
$$

Then $P=\exp (h S)$ satisfies the eq. (2). 


\section{A first proof of Gerstenhaber's formula}

With some more effort we also may prove Gerstenhaber's formula. By (2) we only have to show

$$
\psi\left(E_{1}+E_{2}, D_{3}\right) \psi\left(E_{1}, D_{2}\right)=\psi\left(E_{1}, D_{2}+D_{3}\right) \psi\left(E_{2}, D_{3}\right)
$$

for $\psi(E, D)=P=\left(1+h E_{1}\right)^{D_{2}}$.

To see this lets first collect several helpful identities :

Lemma 1 For $f, g \in K[x][[h]]$ we get

1. $E^{n} f(D)=f(D+n) E^{n}$,

2. $[D, f(E)]=-\left.x \frac{\partial}{\partial x} f(x)\right|_{x=E}$,

3. $f(E) D=\left(D+E \frac{\partial}{\partial E} \ln f(E)\right) \cdot f(E)$,

4. $f(E) g(D)=g\left(D+E \frac{\partial}{\partial E} \ln f(E)\right) \cdot f(E)$ (note that $g\left(D+E \frac{\partial}{\partial E} \ln f(E)\right.$ ) is a function with non commuting arguments!),

5.

$$
e^{h E D}=\sum_{k=0}^{\infty} h^{k} E^{k}\left(\begin{array}{l}
D \\
k
\end{array}\right),
$$

where

$$
\left(\begin{array}{l}
x \\
k
\end{array}\right):=\frac{x(x-1) \ldots(x-k+1)}{k !}, \quad \text { such that }(1+h x)^{y}=\sum_{k=0}^{\infty} h^{k} x^{k}\left(\begin{array}{l}
y \\
k
\end{array}\right) .
$$

6. $f(E) e^{\alpha D}=e^{\alpha D} f\left(e^{\alpha} E\right)$ and $e^{\alpha D} f(E)=f\left(\frac{E}{e^{\alpha}}\right) e^{\alpha D}$.

In particular

7. $(1+h x)^{D} f(E)=f\left(\frac{E}{1+h x}\right)(1+h x)^{D}$.

Proof: These formulas may be proved immediately by straightforward computations. 1.

- 5. follow almost directly from the commutation rule $[E, D]=E$ and linearity. To prove 6. we obtain from 1 . for $f=\sum a_{k} x^{k}$

$$
\begin{gathered}
f(E) e^{\alpha D}=\sum_{k=0}^{\infty} a_{k} E^{k} e^{\alpha D}=\sum_{k=0}^{\infty} a_{k} e^{\alpha(D+k)} E^{k}=\sum_{k=0}^{\infty} a_{k} e^{\alpha D}\left(e^{\alpha k} E^{k}\right) \\
=e^{\alpha D} \sum_{k=0}^{\infty} a_{k}\left(e^{\alpha} E\right)^{k}=e^{\alpha D} f\left(e^{\alpha} E\right) .
\end{gathered}
$$

There is a more rigid result than theorem 2: 
Theorem 5 A power series $f(x, y) \in K[x, y][[h]]$ with $f(0, y)=1, f_{x}(0, y)=h$ y satisfies (马) iff $f=\psi$, i.e.

$$
f(x, y)=(1+h x)^{y}=\sum_{k=0}^{\infty} h^{k} x^{k}\left(\begin{array}{l}
y \\
k
\end{array}\right) .
$$

Proof: Replacing in (3) the commuting variables $E_{1}, D_{3}$ by $x$ resp. $y$ and the remaining non commuting $D_{2}, E_{2}$ by $D, E$ we have to solve the equation

$$
f(x+E, y) f(x, D)=f(x, D+y) f(E, y) .
$$

We will solve this functional equation transforming it to a differential equation for $f$. Take the first derivative with respect to $x$

$$
f_{x}(x+E, y) f(x, D)+f(x+E, y) f_{x}(x, D)=f_{x}(x, D+y) f(E, y)
$$

and set $x=0$. Then $\left(f(0, y)=1, f_{x}(0, y)=h y\right)$

$$
f_{x}(E, y)+f(E, y) h D=h(D+y) f(E, y)
$$

or

$$
f_{x}(E, y)=h[D, f(E, y)]+h y f(E, y)
$$

Lemma 1 yields

$$
[D, f(E, y)]=-E \frac{\partial}{\partial E} f(E, y)=-E f_{x}(E, y) .
$$

Substituting this expression in (4) we get an equation in $E$ only.

$$
f_{x}(E, y)=-h E f_{x}(E, y)+h y f(E, y) \text {. }
$$

Its integral with respect to the initial conditions yields $f(x, y)=(1+h x)^{y}$ and vice versa.

\section{A bialgebra deformation}

An extended version of the condition (2) is contained in the following theorem:

Theorem 6 Assume that the bialgebra $\left(B, \mu_{B}, \Delta_{B}\right)$ acts admissibly on $A$ and $P \in 1+$ $h(B \otimes B)[[h]]$ satisfies condition (2). Then for $A^{\prime}=A[[h]]$ and $B^{\prime}=B[[h]]$

1. $A_{h}=\left(A^{\prime}, \mu_{h}=\mu_{A} \circ P\right)$ is a $K^{\prime}$-algebra.

2. $B_{h}=\left(B^{\prime}, \mu_{B}, \Delta_{h}\right)$ with $\Delta_{h}(b):=P^{-1} \Delta_{B}(b) P$ is a bialgebra.

3. The natural ( $K^{\prime}$-linear) bialgebra action of $B_{h}$ on $A_{h}$ is admissible.

4. $S_{h}=P^{-1} \frac{\partial P}{\partial h}$ is a 2-cocycle of the coalgebra $\left(B^{\prime}, \Delta_{h}\right)$. 
Proof: 2. One has only to prove the coassociativity of $\Delta_{h}$, i.e.

$$
\forall b \in B^{\prime} \quad \Delta_{h, 1} \circ \Delta_{h}(b)=\Delta_{h, 2} \circ \Delta_{h}(b) .
$$

With the definition of $\Delta_{h}$ the left hand side of this expression expands as

$$
P_{12}^{-1} \Delta_{B, 1}\left(P^{-1} \Delta_{B}(b) P\right) P_{12} .
$$

Applying the multiplicativity of $\Delta$ we finally obtain

$$
\left(P_{12}^{-1} \Delta_{B, 1}\left(P^{-1}\right)\right)\left(\Delta_{B, 1}\left(\Delta_{B}(b)\right)\right)\left(\Delta_{B, 1}(P) P_{12}\right)
$$

and analogously for the right hand side

$$
\left(P_{23}^{-1} \Delta_{B, 2}\left(P^{-1}\right)\right)\left(\Delta_{B, 2}\left(\Delta_{B}(b)\right)\right)\left(\Delta_{B, 2}(P) P_{23}\right) .
$$

But the left and right bracket terms are equal by (2) and its inverse whereas the middle bracket terms are the same by the coassociativity of $\Delta_{B}$.

3. We have only to prove that condition (1) is fulfilled, i.e.

$$
b \circ \mu_{h}=b \circ \mu_{A} \circ P=\mu_{h} \circ \Delta_{h}(b)=\mu_{A} \circ \Delta_{B}(b) \circ P .
$$

But this follows immediately from (1) for $B$.

4. From (2) and the definition of $\Delta_{h}$ we obtain

$$
P_{12} \Delta_{h, 1}(P)-P_{23} \Delta_{h, 2}(P)=0 .
$$

Since $\frac{\partial}{\partial h} P=P S_{h}$ the derivative of (2) yields

$$
\Delta_{1}\left(P S_{h}\right) P_{12}+\Delta_{1}(P) P_{12} S_{h, 12}=\Delta_{2}\left(P S_{h}\right) P_{23}+\Delta_{2}(P) P_{23} S_{h, 23} .
$$

Note that further

$$
\Delta_{1}\left(P S_{h}\right) P_{12}=\Delta_{1}(P) \Delta_{1}\left(S_{h}\right) P_{12}=\Delta_{1}(P) P_{12} \Delta_{h, 1}\left(S_{h}\right)
$$

and also

$$
\Delta_{2}\left(P S_{h}\right) P_{23}=\Delta_{2}(P) P_{23} \Delta_{h, 2}\left(S_{h}\right)
$$

Altogether we obtain

$$
\Delta_{1}(P) P_{12} \cdot \delta_{h}(S)=0 .
$$

Hence $\delta_{h}(S)=0$ since the first cofactor is invertible.

This theorem shows that our approach to algebra deformations through admissible bialgebra actions is a very natural one. It does not only allow to formulate a condition on $P$ that implies the associativity of $\mu_{h}=\mu_{A} \circ P$ but also yields a deformation of the coalgebra structure on $B$ in such a way that the deformation process may be iterated. Its this point where we leave the original setting of (universal enveloping algebras of) Lie algebras acting by derivations, since the deformed comultiplication rule is usually more difficult. 
Let's explain these changes on Gerstenhaber's example. For $P=\left(1+h E_{1}\right)^{D_{2}}$ we get as new comultiplication

$$
\Delta_{h}(E)=P^{-1} \Delta_{B}(E) P=\left(1+h E_{1}\right)^{-D_{2}}\left(E_{1}+E_{2}\right)\left(1+h E_{1}\right)^{D_{2}}
$$

Applying the rules collected in lemma 1 we get

$$
\Delta_{h}(E)=E_{1}+E_{2}\left(1+h E_{1}\right)^{-D_{2}+1}\left(1+h E_{1}\right)^{D_{2}}=E_{1}+\left(1+h E_{1}\right) E_{2} .
$$

In the same way we obtain

$$
\Delta_{h}(D)=P^{-1} \Delta(D) P=\left(1+h E_{1}\right)^{-D_{2}} D_{1}\left(1+h E_{1}\right)^{D_{2}}+D_{2} .
$$

Since

$$
\left[\left(1+h E_{1}\right)^{-D_{2}}, D_{1}\right]=E_{1} \frac{\partial}{\partial E_{1}}\left(1+h E_{1}\right)^{-D_{2}}=-h D_{2} E_{1}\left(1+h E_{1}\right)^{-D_{2}-1}
$$

we get

$$
\left(1+h E_{1}\right)^{-D_{2}} D_{1}=\left(D_{1}-h D_{2} E_{1}\left(1+h E_{1}\right)^{-1}\right)\left(1+h E_{1}\right)^{-D_{2}}
$$

and finally

$$
\Delta_{h}(D)=D_{1}+D_{2}-\frac{h E_{1} D_{2}}{\left(1+h E_{1}\right)}=D_{1}+\left(1+h E_{1}\right)^{-1} D_{2} .
$$

Note that moreover

$$
\Delta_{h}(1+h E)=\left(1+h E_{1}\right)\left(1+h E_{2}\right),
$$

i.e. the $B$-cocycle $E$ may be lifted to the $B_{h}$-cocycle $\ln (1+h E)$. Since

$$
S_{h}=P^{-1} \frac{\partial P}{\partial h}=\left(1+h E_{1}\right)^{-D_{2}} \cdot E_{1} D_{2} \cdot\left(1+h E_{1}\right)^{D_{2}-1}=L_{1}^{-1} E_{1} D_{2} .
$$

we get $\delta_{h}(D)=h S_{h}$, i.e. the $B$-cocycle $D$ is not liftable. $S$ is a bialgebra analog of a jump cocycle as defined for algebras in [4].

REmark: Over $K^{\prime}\left[h^{-1}\right]$ the bialgebra $B_{h}$ may be generated by $D$ and $L=1+h E$ with the following relations

$$
\Delta(D)=D_{2}+L_{1}^{-1} D_{2}, \Delta(L)=L_{1} L_{2},[L, D]=L-1 .
$$

There is a $K$-bialgebra $\tilde{B}=K\left\langle D, L, L^{-1}\right\rangle$ with the same relations. If we extend it trivially to $\tilde{B}^{\prime}=\tilde{B}[[h]]$ there is a bialgebra homomorphism

$$
f_{h}: \tilde{B}^{\prime} \longrightarrow B_{h} \quad \text { via } \quad L \mapsto 1+h E .
$$

This $K$-algebra will be considered in the next section. 


\section{Another derivation of Gerstenhaber's formula}

From the above considerations we can extract the conditions on $D, L$ that are necessary for Gerstenhaber's formula to be fulfilled. This way we get the following generalization:

Theorem 7 Let $\tilde{B}$ be a $K^{\prime}$-bialgebra and $L, D \in \tilde{B}$ such that $L^{-1}$ exists and the following relations are fulfilled

$$
L-1 \in h \tilde{B},[L, D]=L-1, \Delta(L)=L_{1} L_{2}, \Delta(L)=D_{1}+L_{1}^{-1} D_{2} .
$$

Then the power series

$$
P=L_{1}^{-D_{2}}=\exp \left(-\ln L_{1} \cdot D_{2}\right)
$$

satisfies eq.(2).

Note that $D$ and $L$ may depend (almost) arbitrarily on $h$.

Proof: For our $P$ eq.(2) has the form

$$
\left(L_{1} L_{2}\right)^{-D_{3}} \cdot L_{1}^{-D_{2}}=L_{1}^{-D_{2}-L_{2}^{-1} D_{3}} \cdot L_{2}^{-D_{3}}
$$

or

$$
L_{1}^{-D_{3}} \cdot L_{2}^{-D_{3}} \cdot L_{1}^{-D_{2}}=L_{1}^{-D_{2}-L_{2}^{-1} D_{3}} \cdot L_{2}^{-D_{3}}
$$

Here only $L_{2}$ and $D_{2}$ don't commute. In order to exchange the two factors $L_{2}^{-D_{3}}$ and $L_{1}^{-D_{2}}$ in the left hand side we introduce the element $E:=L-1$. Then $[E, D]=E$ and by lemma 1 we have

$$
f(E) g(D)=g\left(D+E \frac{\partial}{\partial E} \ln f(E)\right) \cdot f(E)
$$

for $f, g \in K[x][[h]]$. Since

$$
f\left(E_{2}\right)=L_{2}^{-D_{3}}=\left(1+E_{2}\right)^{-D_{3}} \quad \text { and } \quad E_{2} \frac{\partial}{\partial E_{2}} \ln f\left(E_{2}\right)=-E_{2} L_{2}^{-1} D_{3}
$$

the left hand side of (5) may be written as

$$
\left(L_{1}\right)^{-D_{3}} \cdot L_{1}^{-\left(D_{2}-E_{2} L_{2}^{-1} D_{3}\right)} \cdot L_{2}^{-D_{3}} .
$$

Comparing this with the right hand side of (5) we see that the exponents of $L_{1}$ are equal.

Substituting $L=1+h E$ we get a new proof of Gerstenhaber's formula. The special form of $L$ may be derived in the following way: Assume that only $L$ depends on $h$. We get

$$
S_{h}=P^{-1} \frac{\partial P}{\partial h}=L_{1}^{-1} \frac{\partial L_{1}}{\partial h} D_{2}
$$

and the choice of $S_{h}$ as the jump cocycle $\frac{1}{h} \delta D$ gives us

$$
L_{1}^{-1} \frac{\partial L_{1}}{\partial h} D_{2}=\frac{1}{h} L_{1}^{-1}\left(L_{1}-1\right) D_{2},
$$

i.e. $\frac{\partial L}{\partial h}=\frac{1}{h}(L-1)$. Its solution is $L=1+h E$ with $E=\left.L\right|_{h=0}$. 


\section{First order deformations}

In this section let $K^{\prime}=K[h] /\left(h^{2}\right), B^{\prime}=B \otimes_{K} K^{\prime}$, and $P=1 \otimes 1+h S$ for $S \in B^{\prime} \otimes_{K^{\prime}} B^{\prime}$. Our considerations so far may be transferred to this setting to obtain a theory of first order deformations. This linearizes all problems and, e.g., eq. (2) is equivalent to the condition $\delta(S)=0$. Thus there is a one-to-one correspondence between 2-cocycles of the coalgebra $B$ and solutions $P$ of (2).

The new comultiplication in $B_{h}$ defined by theorem 6

$$
\Delta_{h}(b)=P^{-1} \cdot \Delta_{B}(b) \cdot P=(1-h S) \Delta_{B}(b)(1+h S)
$$

yields

$$
\Delta_{h}(b)=\Delta_{B}(b)+h\left[\Delta_{B}(b), S\right]
$$

and for the new coboundary operator $\delta_{h}$ of $B_{h}$

$$
\delta_{h} S=\delta S-h\left[\Delta_{B, 1}(S), S_{12}\right]+h\left[\Delta_{B, 2}(S), S_{23}\right] .
$$

Hence $S$ may not be a $B_{h}$-cocycle. To prolongate the deformation to the next order $S$ has to be changed into $S_{h}=S+h S^{\prime}$ such that

$$
\delta S^{\prime}=\left[\Delta_{B, 1}(S), S_{12}\right]-\left[\Delta_{B, 2}(S), S_{23}\right] .
$$

Let's apply this construction to Gerstenhaber's example. The first order deformation of $B=U(\mathcal{G})$ generated by the 2-cocycle $S=E_{1} D_{2}$ yields the $K[h] /\left(h^{2}\right)$-algebra $B_{h}$ generated by two elements $E, D$ with the following relations

$$
[E, D]=E, \quad \Delta_{h}(E)=E_{1}+E_{2}+h E_{1} E_{2}, \quad \Delta_{h}(D)=D_{1}+D_{2}-h E_{1} D_{2}
$$

and

$$
\delta_{h}(S)=-2 h E_{1} E_{2} D_{3}
$$

For the 2-cochain $E_{1}^{2} D_{2}=E^{2} \otimes D \in B \otimes B$ we get

$$
\delta\left(E^{2} \otimes D\right)=\delta\left(E^{2}\right) \otimes D=-2 E_{1} E_{2} D_{3} .
$$

Thus the $B$-cocycle $S$ may be lifted to the $B_{h}$-cocycle

$$
S_{h}=E_{1} D_{2}-h E_{1}^{2} D_{2}=\left(1-h E_{1}\right) E_{1} D_{2} .
$$

Hence $B_{h}$ has the $K$-bialgebra structure considered in theorem 7 . Thus we derived Gerstenhaber's formula already at the first order deformation step.

\section{References}

[1] V.Coll, M.Gerstenhaber, A.Giaquinto: Explicit formula with non-commuting derivations. In: Ring theory 1989. Proc. Symp. and Workshop, Jerusalem 1988/89. Isr. Math. Conf. Proc. 1 (1989), 396 - 403.

[2] V.G. Drinfel'd: Quantum groups. In: Proc. ICM Berkeley 1986, vol. 1 (1987), 798 -820 . 
[3] M.Gerstenhaber: On the deformation of rings and algebras III. Ann. math. 88 (1968), $1-34$.

[4] M.Gerstenhaber: On the deformation of rings and algebras IV. Ann. math. 99 (1974), $257-276$.

[5] M.Gerstenhaber, A.Giaquito, S.D.Schack: Quantum symmetry. In: Quantum groups. Proc. Workshop Leningrad 1990, Lect. Notes Math. 1510 (1992), 9-46.

[6] D.W. Jonah. Cohomology of coalgebras. Memoires AMS 82 (1968).

[7] J.Moyal: Quantum mechanics as a statistical theory. Proc. Cambrige Phil. Soc. 45 (1949), 99-124.

[8] J.Vey: Déformations du crochet de poisson sur une variété symplectique. Comm. Math. Helv. 50 (1975), 421-454.

[9] A.T.Vlassov: Universal formulas of associative algebra deformation. Advances in Synergetics 5 (1995), 120 - 131.

[10] A.T.Vlassov: Explicit Formulae for Associative Algebra Deformations. Preprint 37/95, NTZ Univ. Leipzig (1995) 